\title{
Studies on the Preparation of Ethylcellulose Microcapsules containing Magnesium Aluminum Hydroxide Hydrate
}

\author{
Syuichi Kasai and Masumi Korshi \\ Faculty of Pharmacentical Sciences, Science University of Tokyol)
}

(Received May 31, 1976)

\begin{abstract}
Ethylcellulose microcapsules containing magnesium aluminum hydroxide hydrate were prepared by the phase separation method using organic polymer solutions and observation on the formation mechanism of wall film during the microencapsulation process was done. tion.

The system of ethylcellulose--dichloromethane- $n$-hexane was used for microencapsula-

In this system, it was found that coacervate drops deposited first on the hydrophobic surface of the core material and the wall was then formed. Furthermore, the region of constant wall thickness was observed irrespective of the weight of ethylcellulose in the microcapsules finally obtained.
\end{abstract}

Keywords-microencapsulation of magnesium aluminum hydroxide hydrate; ethylcellulose coacervate microcapsules; surface appearance; formation mechanism of wall film of microcapsules; wall thickness

In pharmacy, various methods have been used to prepare polymer microcapsules containing drug. Among these techniques, ${ }^{2}$ the phase separation method using coacervation in the system of polymer solutions has generally been used and the detail of the method have been described in many patents, ${ }^{3)}$ and papers. ${ }^{4-6)}$

Cellulose acetate phtharate, ${ }^{4)}$ hydroxypropyl cellulose ${ }^{5)}$ and ethylcellulose ${ }^{6)}$ were used as film-forming agents for encapsulation of solid by simple coacervation. However, the procedural behavior of coacervation has yet been studied in detail.

In this study, therefore, an attempt was done to observe the deposition behavior of ethylcellulose coacervate drops on magnesium aluminum hydroxide hydrate granule, as an antacid drug, by a simple coacervation of the ethylcellulose-dichloromethane- $n$-hexane system at the optimum encapsulation conditions. Furthermore, possible cross sectional models for the deposition of ethylcellulose coacervate drops on the core material and the final state of microcapsule walls were suggested.

\section{Experimental}

Materials_- The core material was magnesium aluminum hydroxide hydrate granule (Kyowa Chemical Industry Co., Ltd.) and had the characteristics given in Table I.

Ethylcellulose $\left(\overline{\mathrm{M}}_{\mathrm{w}}=12000\right.$, reagent grade, Tokyo Kasei Industry Co., Ltd.) was used as the wall material.

Dichloromethane and $n$-hexane, both of which were of reagent grade, were used as the good solvent and nonsolvent (coacervating agent).

All of them were used without purification.

Preparation of Microcapsules-A) Transfering: A series of $20 \mathrm{ml}$ test tubes were taken, each containing $0.250 \mathrm{~g}$ of magnesium aluminum hydroxide hydrate, and to these tubes were added ethylcellulose

1) Location: 12 Funagawara-machi, Ichigaya, Shinjukuku, Tokyo.

2) A. Kondo, "Microcapsule," Nikankogyoshinbunsha, Tokyo, 1970.

3) M. Gutcho, "Capsule Technology and Microencapsulation," Noyes Data Corp., New Jersey, USA, 1972.

4) H.P. Merkle and P. Speiser, J. Pharm. Sci., 62, 1444 (1973).

5) S. Borodkin and F.E. Tucher, J. Pharm. Sci., 63, 1354 (1974).

6) IBM: USP $3,173,878$ (1965). 
TABLE I. Characteristics of Magnesium Aluminum Hydroxide Hydrate

\begin{tabular}{lclc}
\hline \hline MgO content (\%) & 37.4 & $\mathrm{Al}_{2} \mathrm{O}_{3}$ content (\%) & 16.4 \\
$\mathrm{CO}_{2}$ content $(\%)$ & 7.1 & drying loss (\%) & 3.4 \\
pH & 8.7 & specific gravity & 2.05 \\
solubility at $25^{\circ}(\mathrm{ppm})$ & 201 & & \\
granule diameter $(\mathrm{mm})$ & 0.5 & & \\
BET surface area $\left(\mathrm{m}^{2} / \mathrm{g}\right)$ & 110 & & \\
chemical structure: $\mathrm{Mg}_{6} \mathrm{Al}_{2}(\mathrm{OH})_{16} \mathrm{CO}_{3} \cdot 4 \mathrm{H}_{2} \mathrm{O}$ & \\
\hline
\end{tabular}

solution in dichloromethane of concentrations from 0.02 to $10.0 \%(\mathrm{w} / \mathrm{v})$. After $10 \mathrm{minutes}$ standing, $5 \mathrm{ml}$ of distilled water was added to the test tubes and then, the tubes were agitated for 15 seconds by a mixer. After a while, it was observed that the core material existed either in the top or in the bottom phase, which consisted of water and dichloromethane, respectively. After 24 hours standing, the tubes were gently shaken and they allowed to stand age for a week, and the sedimentation volume of the core material was measured in the top and the bottom phases in the tubes, meanwhile, the volume occupied by emulsion was observed in the middle phase.

B) Preparation of Phase Diagram: At $25^{\circ}$, the turbidimeter (Nippon Precision Optical Instrument Co., Ltd., Tokyo, SED-PT-201 type) was used for preparing phase diagram. Various volumes of $n$-hexane were added to ethylcellulose solutions of various concentrations in dichloromethane solution and the turbidities of the mixture were measured immediately after shaking with hand. The point of phase change was taken as the point at which the turbidity started to increase rapidly. The points of phase change at various concentrations of ethylcellulose were determined in this way and the phase diagram was prepared based upon these points of phase change in the solutions.

$n$-Hexane, in the same range of volume described above, was added to ethylcellulose solutions in dichloromethane in a thermostated water bath at $4^{\circ}$ and then, the turbidity was visually observed. Similar observations were made using subdivided $n$-hexane volumes in the range where the added volume caused the turbidity to increase to a visually observable level. The critical weight percentage at the point of phase change was then calculated from the specific gravity data and was plotted on a triangular diagram.

C) Microencapsulation: Ethylcellulose microcapsules of various wall thicknesses were prepared by the following method, as indicated in Chart 1.

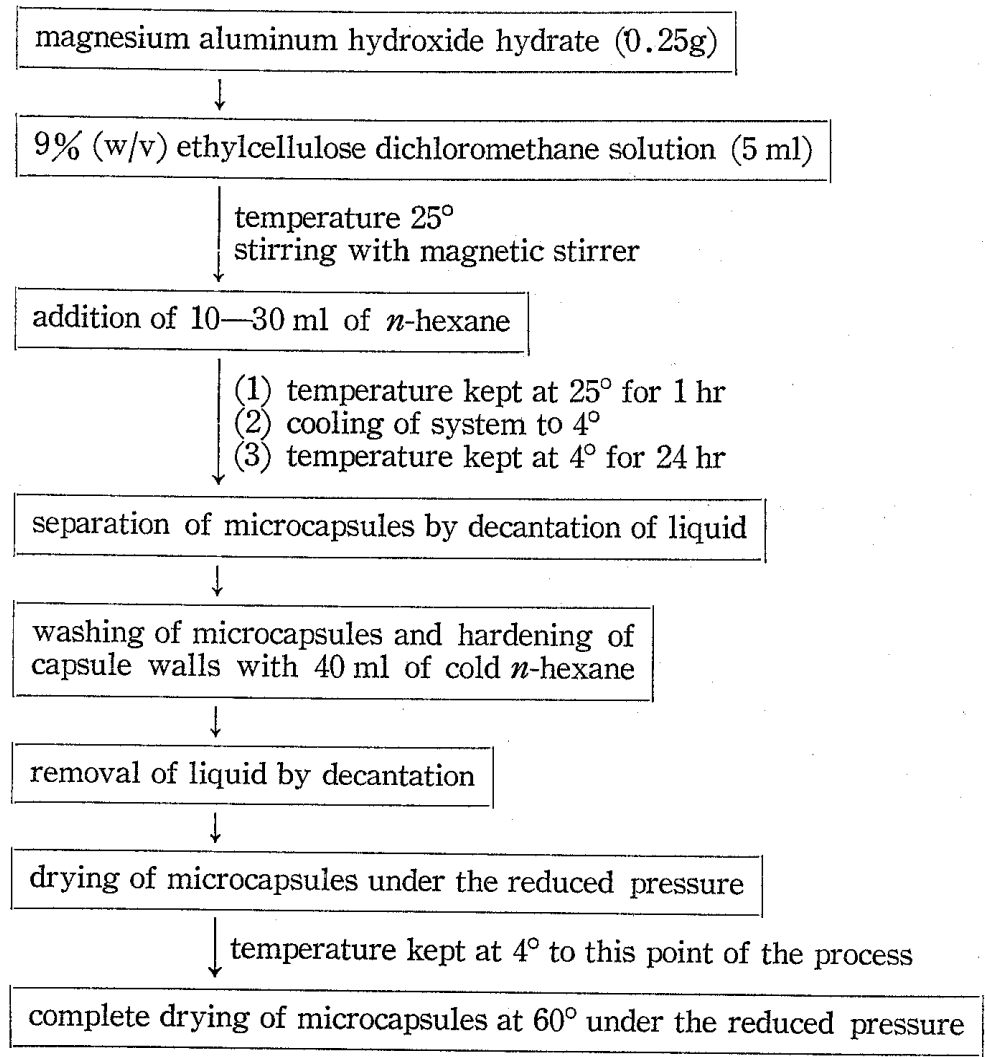

Chart 1. Preparation of Ethylcellulose Microcapsules 
A portion $(0.25 \mathrm{~g})$ of magnesium aluminum hydroxide hydrate, previously dried at $110^{\circ}$ for one hour by an infrared-moisturemeter (Kett Electric Laboratory Co., Ltd., Tokyo, Kett $2 \mathrm{~A}$ type) was added to $5 \mathrm{ml}$ of $9 \%(\mathrm{w} / \mathrm{v})$ ethylcellulose solution in dichloromethane and then, the mixture was stirred for 30 seconds by a magnetic stirrer. An appropriate volume of $n$-hexane was added to it to cause phase separation. The mixture was continuously stirred for one minute, and it was allowed to stand at $25^{\circ}$ for one hour with occasional stirring to promote deposition of coacervate drops on the surface of magnesium aluminum hydroxide hydrate granules. To solidify the wall, the mixture was then permitted to stand at $4^{\circ}$ for $\mathbf{2 4}$ hours. After the supernatant solution was removed by decantation and $40 \mathrm{ml}$ of cold $n$-hexane was added, the mixture was allowed again to stand at $4^{\circ}$ for 24 hours. The supernatant solution was removed, and the precipitate was dried under a reduced pressure at $60^{\circ}$ was required to attain a constant weight of the microcapsules. The amount of phase-separated ethylcellulose could be controlled by adjusting the volume added of $n$-hexane to the system.

D) Determination of Wall Thickness of Microcapsules: The size of microcapsules was measured on about 100 microcapsules under a microscope at 200 magnifications. The wall of the microcapsules was dissolved in dichloromethane and then size of the core material particles was measured by a method similar to that described above after drying. The wall thickness of the microcapsules was thus determined from the difference in the mean size between the microcapsules and the core particles.
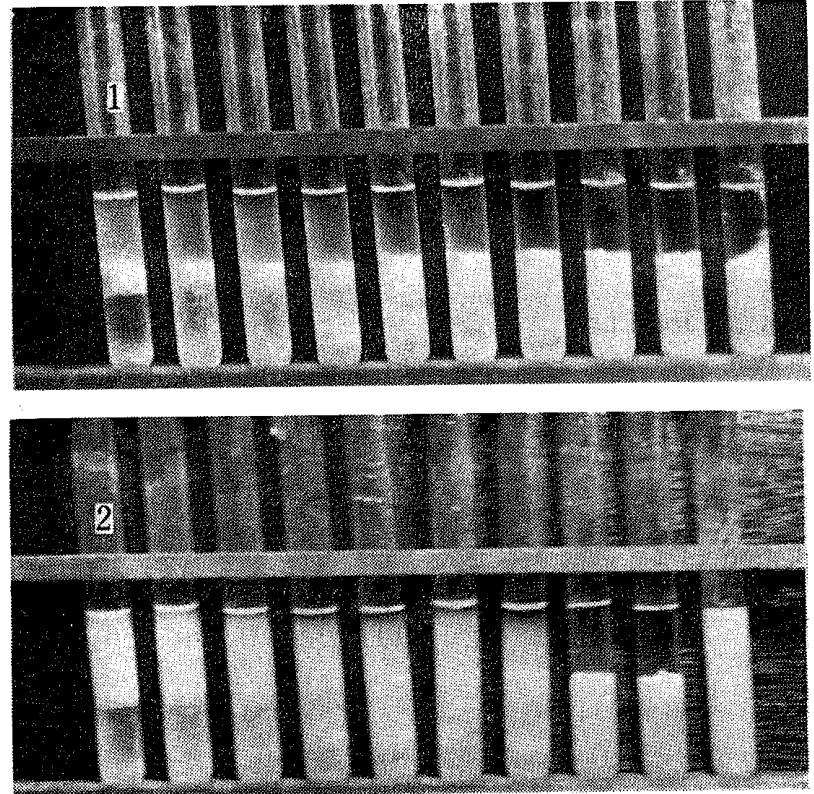

Fig. 2. Transfer States of the Core Material after One Week Standing at $25^{\circ}$

key: (1) one week standing (2) 15 minutes standing, as a reference

note: ethylcellulose concentrations $* 0,0.02,0.05,0.09,0.13$, $0.19,0.35,0.50,0.79,1.0 \%(\mathrm{w} / \mathrm{v}) \quad *$ from left to right sides in figures.

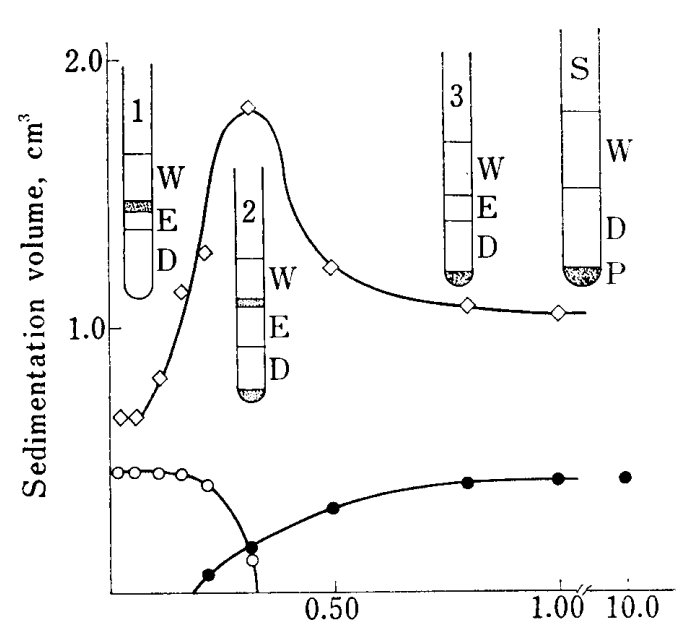

Ethylcellulose concentration, \% $(w / v)$

Fig. 1. Transferability of the Core Material from Ethylcellulose Solution in Dichloromethane to Water at $25^{\circ}$

key: $\bigcirc$, sedimentation volume in water; $\bigcirc$, sedimentation volume in ethylcellulose solution; $\diamond$, sedimentation volume in boundary phase region; $1,2,3$, and $S$ are states of the core material in some experimental conditions. But $S$ is in pretransfering condition; $W$, $\mathrm{E}, \mathrm{D}$, and $\mathrm{P}$, water, boundary phase region, ethylcellulose solution in dichloromethane, and the core material.

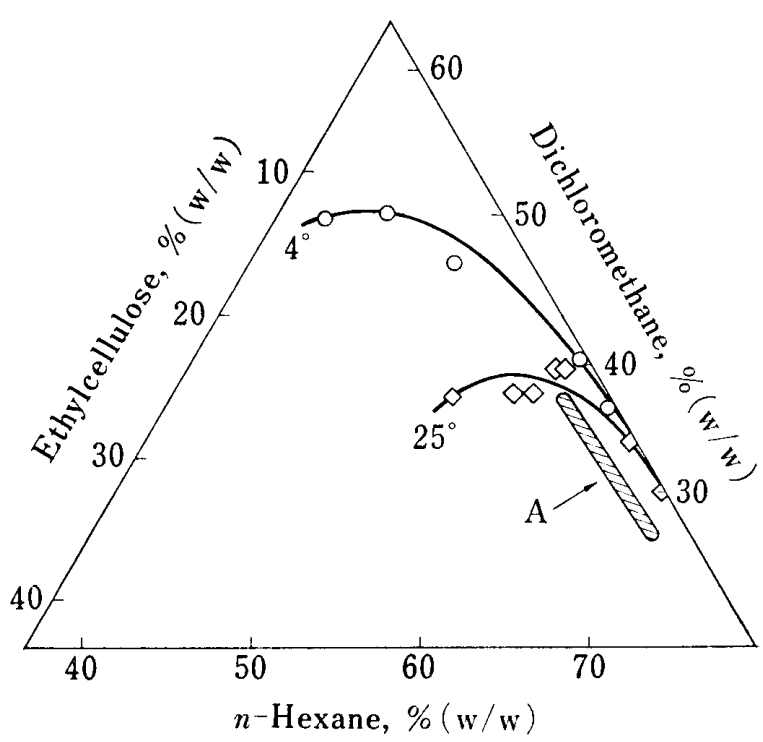

Fig. 3. Triangular Phase Diagram of the Coacervate System, Ethylcellulose-Dichloromethane- $n$-Hexane

key: A, optimum encapsulation region at $25^{\circ}$ 

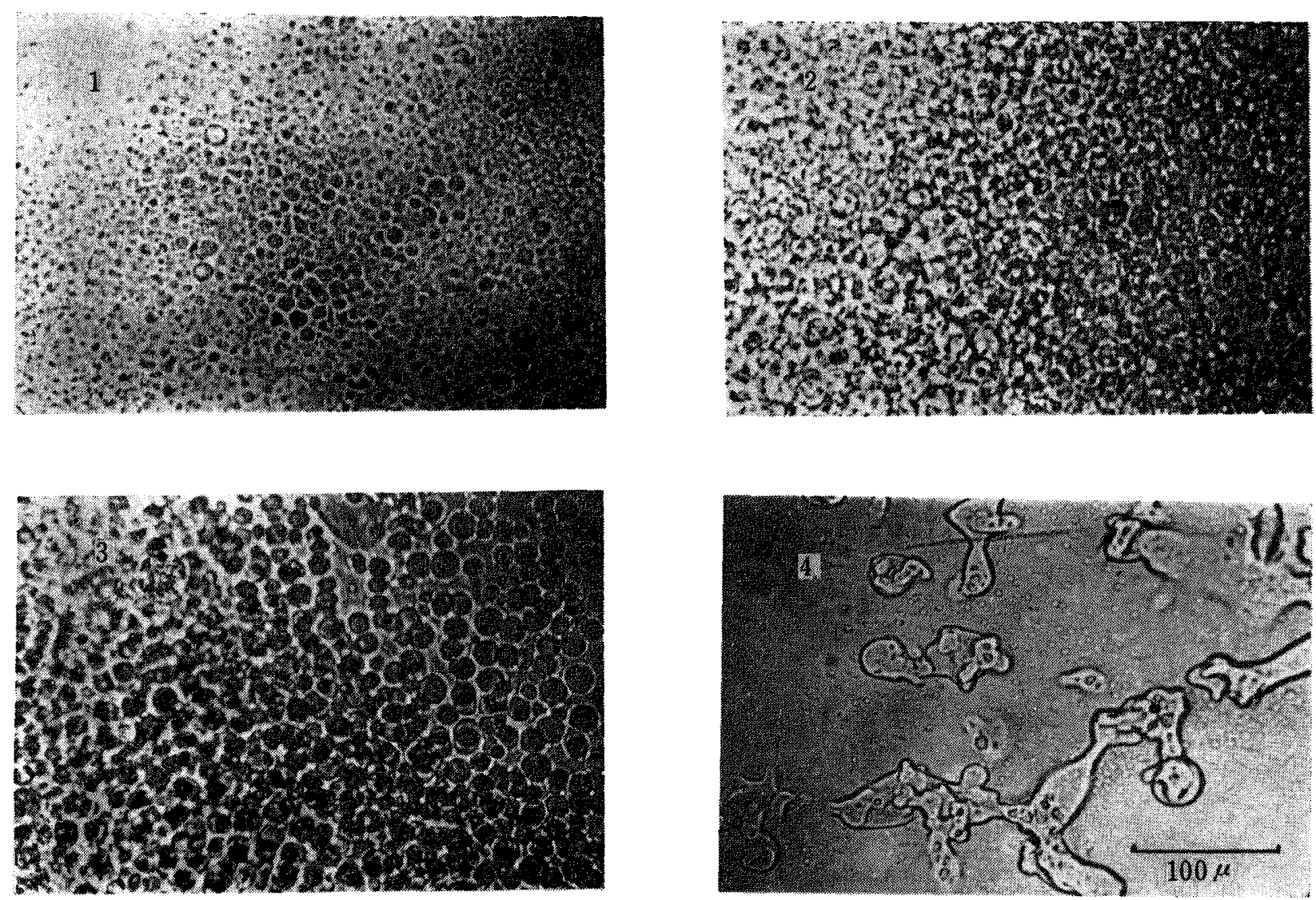

Fig. 4. Photomicrographs of Ethylcellulose Coacervate Drops

key: (1), coacervate in region (1) of Fig. 5; (2) and (3), region (2) in Fig. 5; (4), region (3) in Fig. 5.

Note: (1), $11 \mathrm{ml}$ of $n$-hexane was added in $5 \mathrm{ml}$ of $9 \%(\mathrm{w} / \mathrm{v})$ ethylcellulose solution in dichloromethane; (2), $15 \mathrm{ml}$ of $n$-hexane; (3), $17.5 \mathrm{ml}$ of $n$-hexane; (4), $20 \mathrm{ml}$ of $n$-hexane.

\section{Results and Discussion}

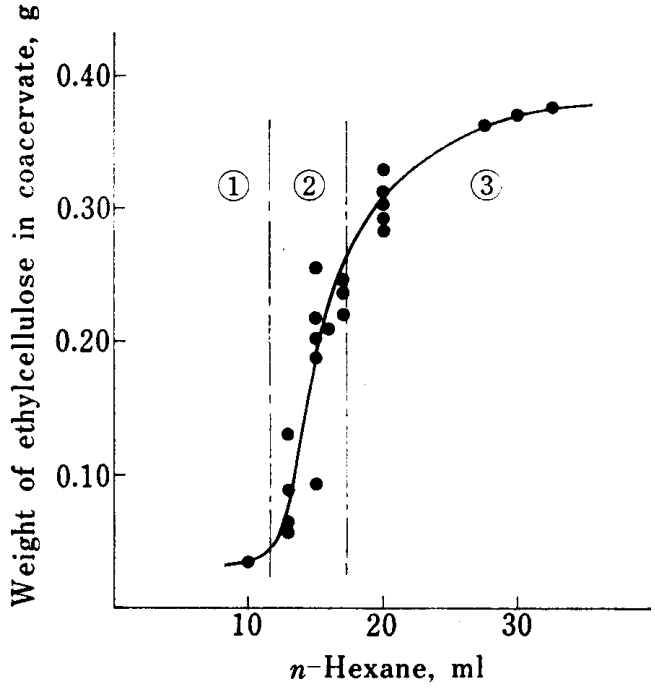

Fig. 5. Weight of Ethylcellulose in Coacervate at $4^{\circ}$ as a Function of Added $n$-Hexane

key: (1), coacervating region in the form of small spherical drops; (2), spherical or ellipsoidal medium size drops; (3), large and irregular shaped drops.

\section{Preparation of Microcapsules}

A) Transferability of Magnesium Aluminum Hydroxide Hydrate to Water Phase__ Figure 1 shows the transfer of magnesium aluminum hydroxide hydrate from dichloromethane to water in the presence of ethylcellulose in the organic phase. As can be seen, there are three regions, 1,2 , and 3 , in the transfer. Most of the core material existed in the water phase while only the trace amount was in an emulsion phase as designated by the region 1 , up to the concentration of $0.25 \%(\mathrm{w} / \mathrm{v})$ ethylcellulose solution in dichloromethane. In the region 2 , where the ethylcellulose concentration lay between 0.30 $0.50 \%(\mathrm{w} / \mathrm{v})$, the core material was distributed in three phases and the transfer of the core material decreased with increasing ethylcellulose concentration. Furthermore, the core material was not in the water phase when the ethylcellulose concentration exceeded $0.50 \%(\mathrm{w} / \mathrm{v})$. 
On the basis of the above mentioned results, the experimental results on the sedimentation volume of the core material in both water and ethylcellulose solution, and the volume occupied by emulsion, which is formed by shaking the core material and two liquids, will be discussed below.

The sedimentation volume in water decreased gradually when the ethylcellulose concentration increased from 0 to $0.35 \%(\mathrm{w} / \mathrm{v})$. On the other hand, the sedimentation volume in ethylcellulose solution increased gradually as the ethylcellulose concentration increased from $0.20 \%(\mathrm{w} / \mathrm{v})$ to $10 \%(\mathrm{w} / \mathrm{v})$. Therefore, the surface of the core material seems to have changed
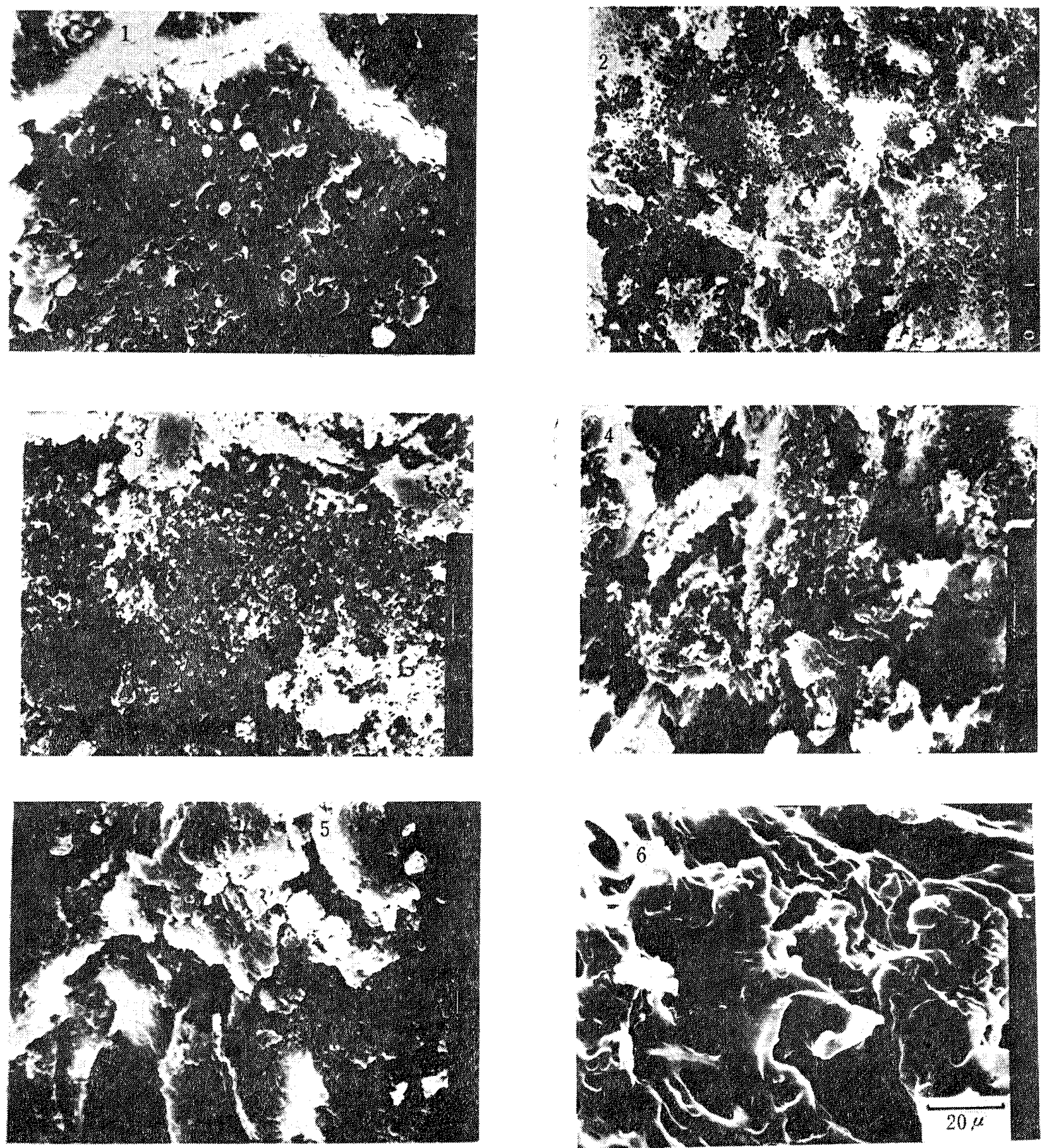

Fig. 6. Scanning Electron Micrographs of Ethylcellulose Microcapsule Surface and Uncoated Core Material Surface

key: (1), uncoated core material; (2), the surface of the core material encapsulated in region (1) of Fig. 5; (3), (4), and (5), region (2) in Fig. 5; (6), region (3) in Fig. 5.

note: (1), uncoated core material; (2), $13 \%(\mathrm{w} / \mathrm{w})$ ethylcellulose concentration in microcapsule finally obtained; (3), $22.5 \%(\mathrm{w} / \mathrm{w}) ;(4), 29 \%(\mathrm{w} / \mathrm{w}) ;(5), 45 \%(\mathrm{w} / \mathrm{w}) ;(6), 60 \%(\mathrm{w} / \mathrm{w})$. 
in stepwise from hydrophilic to hydrophobic one. The change in the surface property was clearly observed when the ethylcellulose concentration was higher than about $0.35 \%(\mathrm{w} / \mathrm{v})$ and it became more distinct if the concentration was above $0.50 \%(\mathrm{w} / \mathrm{v})$.

Figure 2 shows the transfer of the core material in all three regions. The above states after one week standing were the same as those after 24 hours except that the emulsion volume decreased gradually with time. The volume of emulsion in the middle phase, as can be seen in the figure, has a maximum in the boundary region having both hydrophilic and hydrophobic properties.

B) Microencapsulation-The triangular phase diagram of the system ethylcellulosedichloromethane- $n$-hexane is shown in Fig. 3. The region A was used for microencapsulating magnesium aluminum hydroxide hydrate granules, as described in the experimental part $\mathrm{C}$.

Figure 4 shows ethylcellulose coacervate drops formed by the addition of various volumes of $n$-hexane to ethylcellulose solution in dichloromethane. The photographs show that the size and shape of coacervate drops change remarkably with the increase in the amount of $n$ hexane added. We assume that the drops in the photo (4) correspond to those in which phase separation proceeds too much and they cannot be used as coacervate drops to deposit on the core material. When these drops deposit on the hydrophobic surface of magnesium aluminum hydroxide hydrate modified by ethylcellulose adsorption, they can form microcapsule wall having various wall thicknesses.

The correlation of ethylcellulose weight deposited on the surface of the core material and the volume of $n$-hexane added is shown in Fig. 5 . The weight of ethylcellulose in coacervate increased with increasing amount of $n$-hexane showing a sigmoid curve. Subdivided regions 1,2 , and 3 shown in the figure correspond to those in which different volumes of $n$-hexane are needed to form the three kinds of wall having different wall thicknesses discussed elsewhere in this paper. However, the region 3 may not be used to make a suitable wall in microencapsulation, as described in the discussion of the data in Fig. 4.

Figure 6 gives scanning electron micro-

step I

step II

(1)
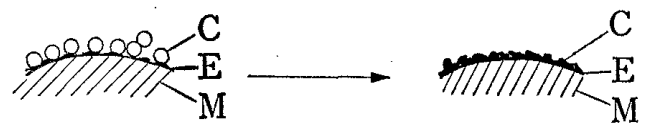

(2)

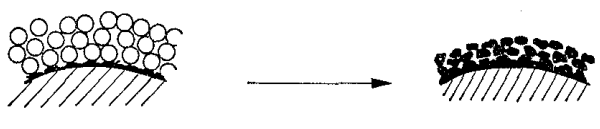

(2)

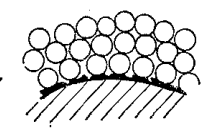

(3)

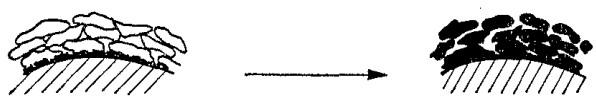

Fig. 7. Possible Cross Sectional Models for the Deposition of Ethylcellulose Coacervate Drops on the Core Material and the Final State of Microcapsule Walls

key: step I: coacervate drops deposited at first; step II: final walls; (1), (2), and (3), see Fig. 4, 5, and 6; C, $E$, and $M$, coacervate drops, ethylcellulose adsorbed initially on the core material, and the core material. note: (2)': the case of more larger coacervate drops than that of (2) in this figure, but it belongs within region (2). graphs of ethylcellulose microcapsules with various weight of the wall material.

The surface (1) is that of an uncoated core granule. The surface (2) has a network structure formed by dried ethylcellulose coacervate. The network structure may be formed by shrinkage of coacervate drops during the drying of the microcapsules. In the case of the surface (3), the surface seems to have formed by further deposition of slightly large coacervate drops onto the network structure of the surface (1). Subsequently, continual changes of the surface appearance were $o b-$ served on the surfaces $(4)-(5)$. The surface somewhat rougher than the surface (3), probably because of changes in the size and shape of coacervate drops as are seen in Fig. 4.

As a result, correlation seems to exist between the appearance of microcapsule walls and the size and shape of deposited coacervate drops.

Figure 7 shows possible cross sectional models for the deposition of ethylcellulose coacervate drops on the core granule (see step 
I) and the final wall films of the microcapsules (see step II). The models in both steps were postulated on the basis of the microphotographs of coacervate drops (Fig. 4) and the scanning electron micrographs of the microcapsule surface (Fig. 6).

The wall thickness of the microcapsules prepared by the method described in Fig. 7 is given in Table II.

TABLE II. Effect of Ethylcellulose Concentration on Wall Thickness

\begin{tabular}{llrrrrrrrr}
\hline \hline Ethylcellulose concentration ${ }^{a)} \%(\mathrm{w} / \mathrm{w})$ & 8.7 & 10.8 & 27.2 & 30.4 & 36.1 & 45.0 & 52.8 & 57.7 \\
Wall thickness $(\mu)$ & 6.2 & 7.5 & 15.0 & 16.2 & 17.5 & 16.2 & 21.2 & 36.2 \\
\hline
\end{tabular}

a) in microcapsule finally obtained

It is observed that the wall thickness is in the range $6.2-36.2 \mu$ and has the almost same value when the microcapsules are prepared using $27-4.6 \%(\mathrm{w} / \mathrm{w})$ ethylcellulose concentration in microcapsules finally obtained. These results correspond with that of possible cross sectional models in final walls in Fig. 7.

Acknowledgements The authors thank Miss Ihoe Kusunoki of the Tokyo Metropolitan Industrial Technology Center for her assistance to photograph the microcapsules by scanning electron microscope. The investigation was supported by a Scientific Research Grant from the Ministry of Education, Science and Culture Japan (No. 011004). 